Supporting Information for

\title{
Time-Programmed Peptide Helix Inversion of a Synthetic Metal Complex Triggered by an $\mathrm{Achiral}^{\mathrm{NO}_{3}}{ }^{-}$Anion
}

Hiroyuki Miyake,* Hiroshi Kamon, Ikuko Miyahara, Hideki Sugimoto, Hiroshi Tsukube ${ }^{1}$

Department of Chemistry, Graduate School of Science, Osaka City University

Sugimoto, Sumiyoshi-ku, Osaka 558-8585, Japan

* To whom corresponding should be addressed. E-mail: miyake@sci.osaka-cu.ac.jp 


\section{Table of Contents}

1. Measurements

2. Synthesis of ligand $\mathbf{L} \mathbf{2}$

3. Crystallographic Studies

4. References

5. Figures and Table

Figure S1. CD spectra of $\mathbf{L} 2$ and $\mathbf{L} 2-\mathrm{Co}\left(\mathrm{ClO}_{4}\right)_{2}$ complex.

Figure S2. Solvent dependence of NH signals of $\mathbf{L} 2$.

Figure S3. Temperature dependence of NH signals of $\mathbf{L} 2$.

Figure S4. NOESY spectra of $\mathbf{L} 2$.

Figure S5. ORTEP drawing of $\mathbf{L} 2-\mathrm{Zn}\left(\mathrm{ClO}_{4}\right)_{2}$ and $\mathbf{L} 2-\mathrm{Zn}\left(\mathrm{NO}_{3}\right)_{2}$ complexes.

Figure S6. Solvent dependence of $\mathbf{L 2}-\mathrm{Zn}\left(\mathrm{ClO}_{4}\right)_{2}$ complex.

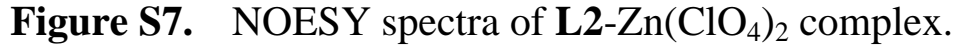

Figure S8. Solvent dependence of $\mathbf{L} 2-\mathrm{Zn}\left(\mathrm{NO}_{3}\right)_{2}$ complex.

Figure S9. NOESY spectra of $\mathbf{L} 2-\mathrm{Zn}\left(\mathrm{NO}_{3}\right)_{2}$ complex.

Figure S10. CD spectra of $\mathbf{L 2 - M}\left(\mathrm{ClO}_{4}\right)_{2}$ complexes in the presence and absence of $\mathrm{NO}_{3}^{-}$.

Figure S11. ESI-MS spectra of $\mathbf{L 2 - M}\left(\mathrm{ClO}_{4}\right)_{2}$ complexes.

Table S1. Torsion angels of peptide chains in $\mathbf{L} \mathbf{2}-\mathrm{Zn}\left(\mathrm{ClO}_{4}\right)_{2}$ and $\mathbf{L} \mathbf{2}-\mathrm{Zn}\left(\mathrm{NO}_{3}\right)_{2}$ complexes. 


\section{Measurements}

${ }^{1} \mathrm{H}$ and ${ }^{13} \mathrm{C}$ NMR spectra were recorded on Jeol LA-300 or -400 FT-NMR spectrometer at ca. $25^{\circ} \mathrm{C}$. NOESY spectra were obtained on Bruker AVANCE 600. IR spectra were obtained with a PerkinElmer Spectrum One FT-IR spectrophotometer. Mass spectra were obtained with Jeol AX-500 or Jeol JMS-700T spectrometer. Polarization degree and melting points were recorded with a Jasco DIP-370 and Yanako MP-J3, respectively. UVvis and CD spectra were recorded on a HITACHI U-3500L and a JASCO J-820 spectrophotometer. The stopped flow CD experiments were performed by a JASCO J-820 spectrophotometer with SFA-452 equipment at $c a .25^{\circ} \mathrm{C}$. The metal complexes for NMR, UV-Vis and CD studies were generated in situ by mixing each metal salt and ligand under conditions shown in each figure caption.

\section{Synthesis of ligand L2}

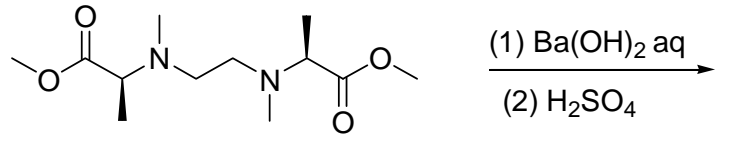

1

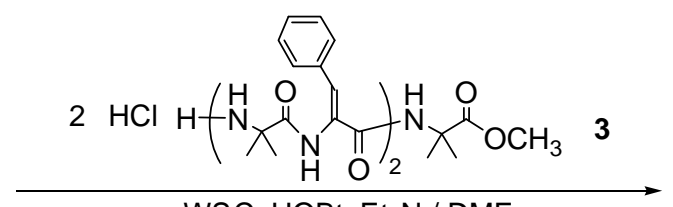

WSC, $\mathrm{HOBt}, \mathrm{Et}_{3} \mathrm{~N} / \mathrm{DMF}$

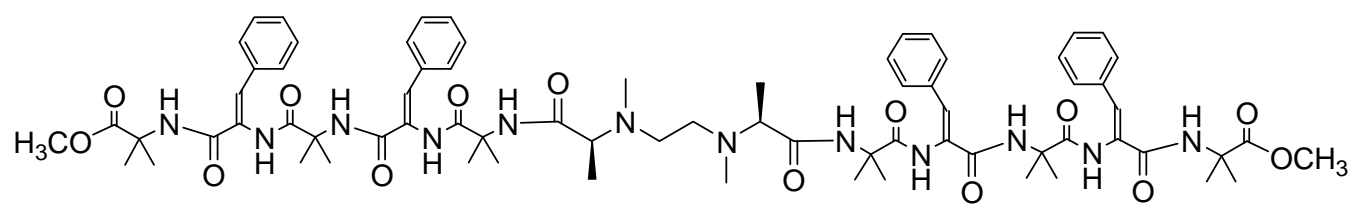

MeO-Aib(5)- $\Delta$ Phe(4)-Aib(3)- $\Delta$ Phe(2)-Aib(1)-Ala-en-Ala-Aib(1)- $\Delta$ Phe(2)-Aib(3)- $\Delta$ Phr(4)-Aib(5)-OMe

\section{(1) $N, N^{\prime}$ - Ethylene-bis-( $N$-methyl-L-alanine) 2}

To a solution of $N, N^{\prime}$-dimethyl- $N, N^{\prime}$-ethylene-bis(L-alanine methyl ester) $\mathbf{1}^{\mathrm{S} 1)}$ (3.58g, $13.7 \mathrm{mmol})$ in $70 \mathrm{ml}$ of $\mathrm{MeOH}$, a solution of $\mathrm{Ba}(\mathrm{OH})_{2} \cdot 8 \mathrm{H}_{2} \mathrm{O}(5.25 \mathrm{~g}, 16.6 \mathrm{mmol})$ in $80 \mathrm{ml}$ of $\mathrm{H}_{2} \mathrm{O}$ was added at room temperature and the solution was stirred for $3 \mathrm{~h}$. $10 \mathrm{ml}$ of $1.67 \mathrm{M}$ 
$\mathrm{H}_{2} \mathrm{SO}_{4}$ aqueous solution was added. After removal of white precipitate by filtration, the filtrate was concentrated by evaporation. Addition of ethanol to the aqueous solution of product in minimal volume precipitated white powder (yield 2.81g, 12.1mmol, 88.2\%). Found: C, 45.75; H, 8.94; $\mathrm{N}, 10.69 \%, \mathrm{C}_{10} \mathrm{H}_{20} \mathrm{~N}_{2} \mathrm{O}_{4} \cdot 1.7 \mathrm{H}_{2} \mathrm{O}$ (hygroscopic) requires C, 45.69; H, 8.97; N, 10.66\%. mp = 147-149 C. FAB MS m/z = $231\left([\mathrm{M}-\mathrm{H}]^{-}\right)$. IR(KBr) 3447 $\left(v_{\mathrm{C}=0}\right) .[\alpha]_{\mathrm{D}}{ }^{30}=24^{\circ}$ (c=0.51, $1 \mathrm{M} \mathrm{HCl}$ aq.). ${ }^{1} \mathrm{H} \operatorname{NMR}\left(\mathrm{D}_{2} \mathrm{O}\right) \delta 1.55$ (d, $\left.6 \mathrm{H}, J=7.3, \beta \mathrm{CH}_{3}\right)$, 2.95 (s, 6H, $\mathrm{NCH}_{3}$ ), 3.5-3.7 (m, 4H, ethylene), 3.99 (q, 2H, J=7.3, $\left.\alpha \mathrm{CH}\right) .{ }^{13} \mathrm{C} \mathrm{NMR}\left(\mathrm{D}_{2} \mathrm{O}\right.$ containing minimum amount of dioxane as internal standard, 67.19ppm): $\delta 11.7\left(\beta \mathrm{CH}_{3}\right)$, 39.7 $\left(\mathrm{NCH}_{3}\right), 51.0$ (ethylene), $66.6(\alpha \mathrm{CH}), 174.3(\mathrm{C}=\mathrm{O})$.

\section{(2) $N$, $N$ '- Ethylene-bis-( $N$-methyl-L-Ala-(Aib- $\Delta$ Phe) $)_{2}$-Aib methylester) L2}

A solution of bisamino acid $2(0.555 \mathrm{~g}, 2.39 \mathrm{mmol})$ and 1-hydoxybenzotriazole hydrate $\left(\mathrm{HOBt} \cdot \mathrm{H}_{2} \mathrm{O}\right)(0.805 \mathrm{~g}, 5.25 \mathrm{mmol})$ in $30 \mathrm{ml}$ of $\mathrm{N}, \mathrm{N}$-dimethylformamide (DMF) was stirred for $10 \mathrm{~min}$ at room temperature and cooled down to $0^{\circ} \mathrm{C}$. A solution of 1-ethyl-3-(3dimethylaminopropyl)carbodiimide hydrochloride (1.01g, 5.25mmol) in 50ml of DMF which was pre-cooled in freezer to $-10^{\circ} \mathrm{C}$ was added and stirred for $30 \mathrm{~min}$ at $0^{\circ} \mathrm{C}$. Penta-

peptide $3^{\mathrm{S} 2}$ (3.35g, $5.26 \mathrm{mmol}$ ) and triethyamine (0.581g, $5.74 \mathrm{mmol}$ ) were added and then the mixture was stirred for 2 days, during which the solution raised up to room temperature. The solvent was removed in vacuo and the residue was dissolved in $30 \mathrm{ml}$ of chloroform and washed by saturated $\mathrm{NaHCO}_{3}$ aqueous solution $(10 \mathrm{ml} \times 3)$, then the organic phase was dried over anhydrous $\mathrm{Na}_{2} \mathrm{SO}_{4}$. The solution was evaporated, and the product was isolated by chromatography on silica gel (160g) with toluene/ethylacetate/methanol (5/4/1) eluent and was further purified from acetonitrile/chloroform/diethyl ether solution to give white powder (yield 319mg, 0.24mmol, 9.9\%). Found: C 63.77; H 6.99; N 12.37\%, $\mathrm{C}_{72} \mathrm{H}_{94} \mathrm{~N}_{12} \mathrm{O}_{14}$ requires C 63.98; H 7.01; N 12.44\%. $\mathrm{mp}=220-221^{\circ} \mathrm{C}$. FAB MS $m / z=1351.7[\mathrm{M}+\mathrm{H}]^{+}$. $\operatorname{IR}(\mathrm{KBr}) 1664,1630,1534\left(v_{\mathrm{C}=0}\right) .[\alpha]_{\mathrm{D}}{ }^{21}=35^{\circ}\left(\mathrm{c}=0.89, \mathrm{CH}_{3} \mathrm{CN}\right) .{ }^{1} \mathrm{H} \mathrm{NMR}\left(\mathrm{CD}_{3} \mathrm{CN}\right): \delta 1.13$ (d, 6H, J=6.96, Ala- $\mathrm{CH}_{3}$ ), 1.36 (s, 6H, Aib-CH $), 1.40$ (s, 6H, Aib-CH CH $_{3} 1.445$ (s, 6H, Aib$\mathrm{CH}_{3}$ ), 1.448 (s, 6H, Aib-CH $\mathrm{CH}_{3}, 1.53$ (s, 6H, Aib-CH $\mathrm{CH}_{3}, 1.55$ (s, 6H, Aib-CH $\mathrm{CH}_{3}, 2.22$ (s, 6H, N-CH ), 2.48 (m, 4H, ethylene), 3.24 (q, 2H, $J=6.96$, Ala- $\alpha \mathrm{H}), 3.59$ (s, 6H, Aib-OCH $)_{3}$, 7.27-7.55 (overlap, 24H, $\Delta$ Phe-phenyl $+\mathrm{C}^{\beta}-\mathrm{H}$ ), 7.83 (s, 1H, NH), 7.86 (s, 1H, NH), 8.26 (s, 
1H, NH), 8.27 (s, 1H, NH), $8.58(\mathrm{~s}, 1 \mathrm{H}, \mathrm{NH}) .{ }^{13} \mathrm{C}$ NMR $\left(\mathrm{CD}_{3} \mathrm{CN}\right): \delta 9.64\left(\mathrm{Ala}-\beta \mathrm{CH}_{3}\right), 24.5$ (Aib- $\left.\beta \mathrm{CH}_{3}\right), 25.1\left(\mathrm{Aib}-\beta \mathrm{CH}_{3}\right), 25.2\left(\mathrm{Aib}-\beta_{\mathrm{CH}}\right), 25.6\left(\mathrm{Aib}-\beta \mathrm{CH}_{3}\right), 25.8\left(\mathrm{Aib}-\beta_{\mathrm{CH}}\right), 26.0$ (Aib- $\beta_{\mathrm{CH}_{3}}$ ), $39.7\left(\mathrm{~N}-\mathrm{CH}_{3}\right.$ ), 52.5 (Aib-OCH${ }_{3}$ ), 52.8 (ethylene), 56.8 (Aib- $\alpha \mathrm{C}$ ), 57.4 (Aib$\alpha \mathrm{C}$ ), 58.4 (Aib- $\alpha \mathrm{C}$ ), 63.7 (Ala- $\alpha \mathrm{C}$ ), 129.4 ( $\Delta$ Phe-phenyl or - $\beta \mathrm{C}$ ), 129.6 ( $\Delta$ Phe-phenyl or $\beta C), 129.8$ ( $\Delta$ Phe-phenyl or $-\beta C$ ), 129.9 ( $\Delta$ Phe-phenyl or $-\beta C$ ), 130.1 ( $\Delta$ Phe-phenyl or $-\beta C$ ), 130.2 ( $\Delta$ Phe-phenyl or - $\beta C$ ), 130.48 ( $\Delta$ Phe-phenyl or $-\beta C), 130.52$ ( $\Delta$ Phe-phenyl or $-\beta C)$, 131.0 ( $\Delta$ Phe-phenyl or - $\beta C$ ), 132.4 ( $\Delta$ Phe-phenyl or $-\beta C), 134.9(\Delta$ Phe- $\alpha \mathrm{C}), 135.2(\Delta$ Phe$\alpha \mathrm{C}), 164.8(\mathrm{C}=\mathrm{O}), 166.6(\mathrm{C}=\mathrm{O}), 175.1(\mathrm{C}=\mathrm{O}), 175.7(\mathrm{C}=\mathrm{O}), 176.0(\mathrm{C}=\mathrm{O}), 176.7(\mathrm{C}=\mathrm{O})$.

\section{Crystallographic Studies}

(1) $\left[\mathrm{Zn}(\mathbf{L 2})\left(\mathrm{H}_{2} \mathrm{O}\right)_{2}\right]\left(\mathrm{ClO}_{4}\right)_{2}\left(\mathrm{CH}_{3} \mathrm{CN}\right)_{0.5}\left(\mathrm{H}_{2} \mathrm{O}\right)_{10}$

Crystal suitable for X-ray analysis was obtained from a solution of ligand $\mathbf{L} 2$ and $\mathrm{Zn}\left(\mathrm{ClO}_{4}\right)_{2} \cdot 6 \mathrm{H}_{2} \mathrm{O}$ in acetonitrile. X-ray diffraction data were collected on colourless crystals of $\left[\mathrm{Zn}(\mathbf{L} 2)\left(\mathrm{H}_{2} \mathrm{O}\right)_{2}\right]\left(\mathrm{ClO}_{4}\right)_{2}\left(\mathrm{CH}_{3} \mathrm{CN}\right)_{0.5}\left(\mathrm{H}_{2} \mathrm{O}\right)_{10}$ with $0.4 \times 0.2 \times 0.2 \mathrm{~mm}$ by Rigaku / MSC Mercury CCD diffractometer with graphite monochromated Mo K $\alpha$ radiation $(\lambda=0.71070$ $\AA \AA$ ) to $2 \theta$ max of $55.0^{\circ}$. Data were processed on a PC using CrystalClear software (Rigaku). The crystal structures were solved by Direct methods (Sir-2004) ${ }^{\mathrm{S} 3)}$ and refined by fullmatrix least squares on $\mathrm{F}^{2}$ using SHELXL-97. ${ }^{\text {S4) }}$ All hydrogen atoms were placed on ideally geometrical positions. Non-hydrogen atoms were refined anisotropically, except for the disordered atoms. Absolute configuration of the complex was determined by the configuration of ligand and Flack parameter. The crystal includes a lot of disordered water molecules. The large value of $\mathrm{R}_{1}$ and $\mathrm{wR}_{2}$ are common for supramolecular crystal included a large number of disordered atoms. Due to stereochemical restraint refinement of $\mathrm{ClO}_{4}^{-}$ molecules, the value of max/min residual density is large.

\section{(2) $\left[\mathrm{Zn}(\mathbf{L} 2)\left(\mathrm{NO}_{3}\right)\right]\left(\mathrm{NO}_{3}\right)\left(\mathrm{CH}_{3} \mathrm{CN}\right)\left(\mathrm{CHCl}_{3}\right)_{5}$}

Crystal suitable for X-ray analysis was obtained from a acetonitrile solution of ligand $\mathbf{L} 2$ and $\mathrm{Zn}\left(\mathrm{NO}_{3}\right)_{2} \cdot 6 \mathrm{H}_{2} \mathrm{O}$ by vapour diffusion of chloroform. X-ray diffraction data were 
collected on colourless crystals of $\left[\mathrm{Zn}(\mathbf{L} 2)\left(\mathrm{NO}_{3}\right)\right]\left(\mathrm{NO}_{3}\right)\left(\mathrm{CH}_{3} \mathrm{CN}\right)\left(\mathrm{CHCl}_{3}\right)_{5}\left(\mathrm{H}_{2} \mathrm{O}\right)$ with $0.2 \times$ $0.2 \times 0.2 \mathrm{~mm}$ by Rigaku / MSC Mercury CCD diffractometer with graphite monochromated Mo K $\alpha$ radiation ( $\lambda=0.71070 \AA$ ) to $2 \theta$ max of $55.0^{\circ}$. Data were processed on a PC using CrystalClear software (Rigaku). The crystal structures were solved by Direct methods (Sir2004) ${ }^{\mathrm{S3})}$ and refined by full-matrix least squares on $\mathrm{F}^{2}$ using SHELXL-97. ${ }^{\mathrm{S4})}$ All hydrogen atoms were placed on ideally geometrical positions. Non-hydrogen atoms were refined anisotropically, except for the disordered atoms of solvents. Absolute configuration of the complex was determined by the configuration of ligand and Flack parameter.

\section{References}

S1. $\quad$ Miyake, H.; Yoshida, K.; Sugimoto, H.; Tsukube, H. J. Am. Chem. Soc. 126, 6524 (2004).

S2. Inai, Y.; Kurokawa, Y.; Hirabayashi, T. Biopolymers 49, 551 (1999).

S3. Burla, M.C.; Caliandro, R.; Camalli, M.; Carrozzini, B.; Cascarano, G.L.; De Caro, L.; Giacovazzo, C.; Polidori, G.; Spagna, R. J. Appl. Cryst. 38, 381 (2005).

S4. Sheldrick, G. M. SHELXL-97; University of Göttingen: Göttingen, Germany, 1997. 


\section{5. $\quad$ Figures and Table}

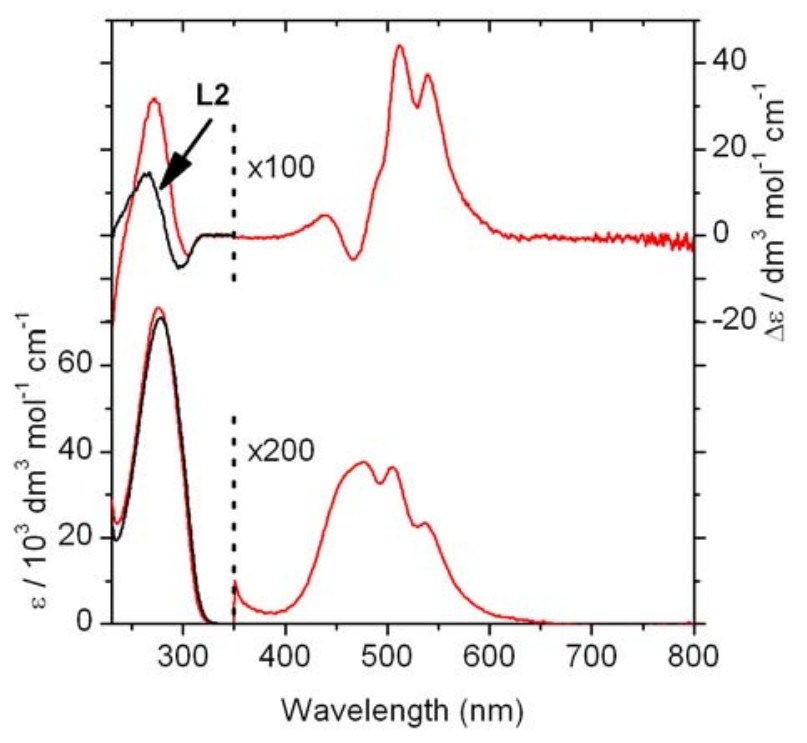

Figure S1. CD (upper) and absorption (lower) spectra of L2 (black) and 1:1 mixture of L2 and $\mathrm{Co}\left(\mathrm{ClO}_{4}\right)_{2} \cdot 6 \mathrm{H}_{2} \mathrm{O}$ in $\mathrm{CH}_{3} \mathrm{CN}$ at room temperature. [L2] $=\left[\mathrm{Co}\left(\mathrm{ClO}_{4}\right)_{2} \cdot 6 \mathrm{H}_{2} \mathrm{O}\right]=1.5 \times 10^{-3}$ mol dm${ }^{-3}, 0.1 \mathrm{~mm}$ cuvette for $<350 \mathrm{~nm}, 10 \mathrm{~mm}$ cuvette for $>350 \mathrm{~nm}$. $\Delta \varepsilon$ values were calculated per ligand concentration. 


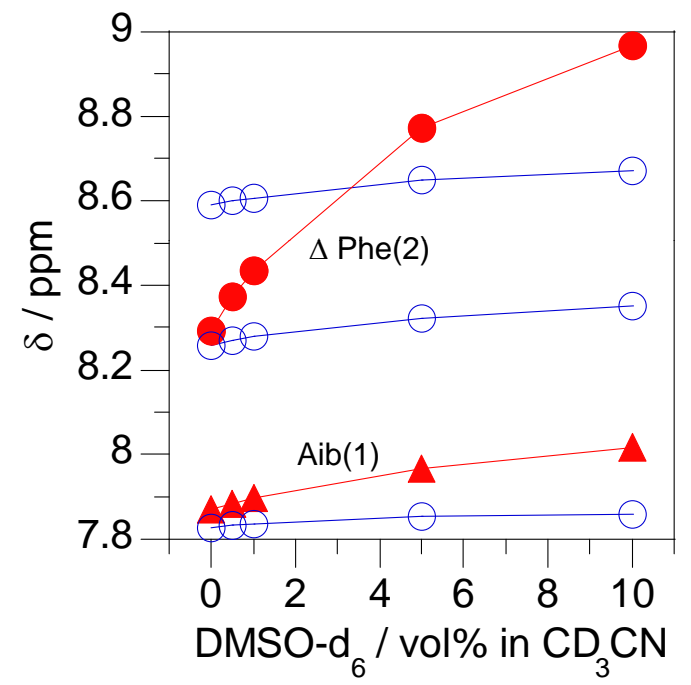

Figure S2. Solvent dependence of NH chemical shifts of L2 in DMSO-d 6 / $\mathrm{CD}_{3} \mathrm{CN}$ mixture system at R.T. $[\mathbf{L 2}]=5 \times 10^{-3} \mathrm{~mol} \mathrm{dm}^{-3}$.

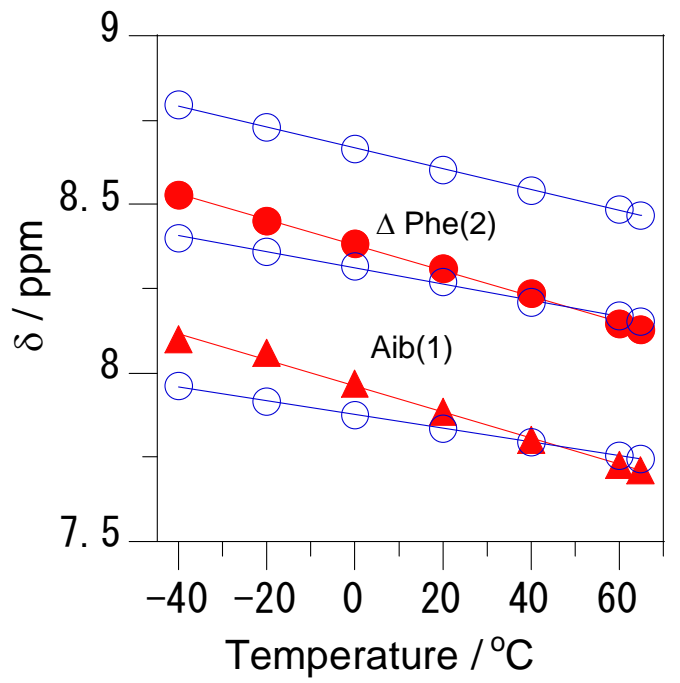

Figure S3. Temperature dependence of $\mathrm{NH}$ chemical shifts of $\mathbf{L 2}$ in $\mathrm{CD}_{3} \mathrm{CN}$. $[\mathbf{L} 2]=5 \times 10^{-3} \mathrm{~mol} \mathrm{dm}^{-3}$. 


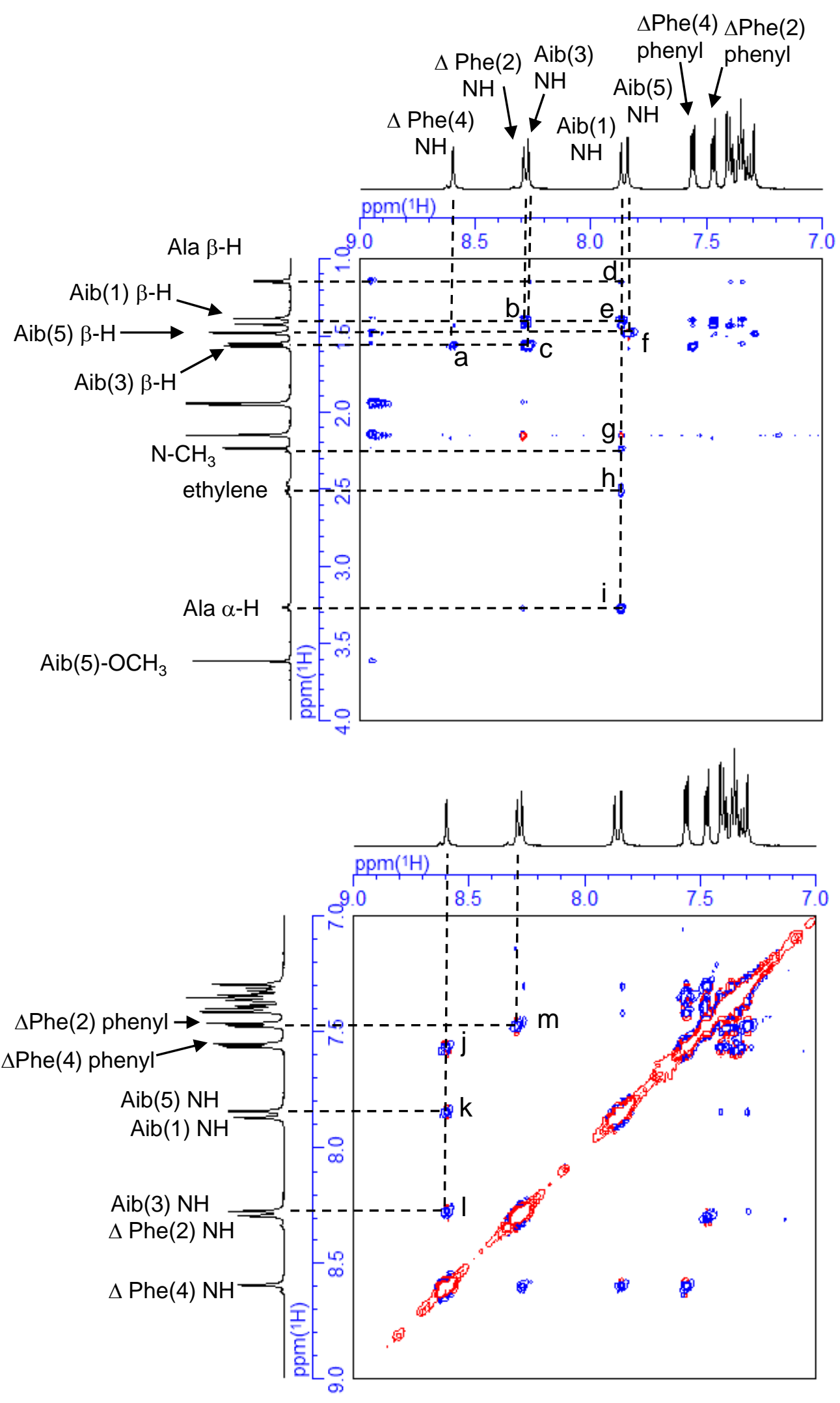

Figure S4. NOESY spectra of $\mathbf{L} 2$ in $\mathrm{CD}_{3} \mathrm{CN}$ at room temperature, $[\mathbf{L 2}]=5 \times 10^{-3} \mathrm{~mol} \mathrm{dm}^{-3}$. a: Aib(3) $\beta-H-\Delta \mathrm{Phe}(4) \mathrm{NH}, \mathrm{b}: \mathrm{Aib}(1) \beta-\mathrm{H}-\Delta \mathrm{Phe}(2) \mathrm{NH}, \mathrm{c}: \mathrm{Aib}(3) \beta-\mathrm{H}-\mathrm{Aib}(3) \mathrm{NH}$, d: Ala $\beta-H-A i b(1) N H$, e: Aib(1) $\beta-H-A i b(1) N H$, f: Aib(5) $\beta-H-A i b(5) N H$, g: $\mathrm{N}-\mathrm{CH}_{3}-\mathrm{Aib}(1) \mathrm{NH}$, h: ethylene-Aib(1) NH, i: Ala $\alpha-\mathrm{H}-\mathrm{Aib}(1) \mathrm{NH}$,

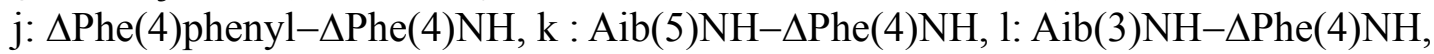
$\mathrm{m}: \Delta \mathrm{Phe}(2)$ phenyl- $\Delta \mathrm{Phe}(2) \mathrm{NH}$, 

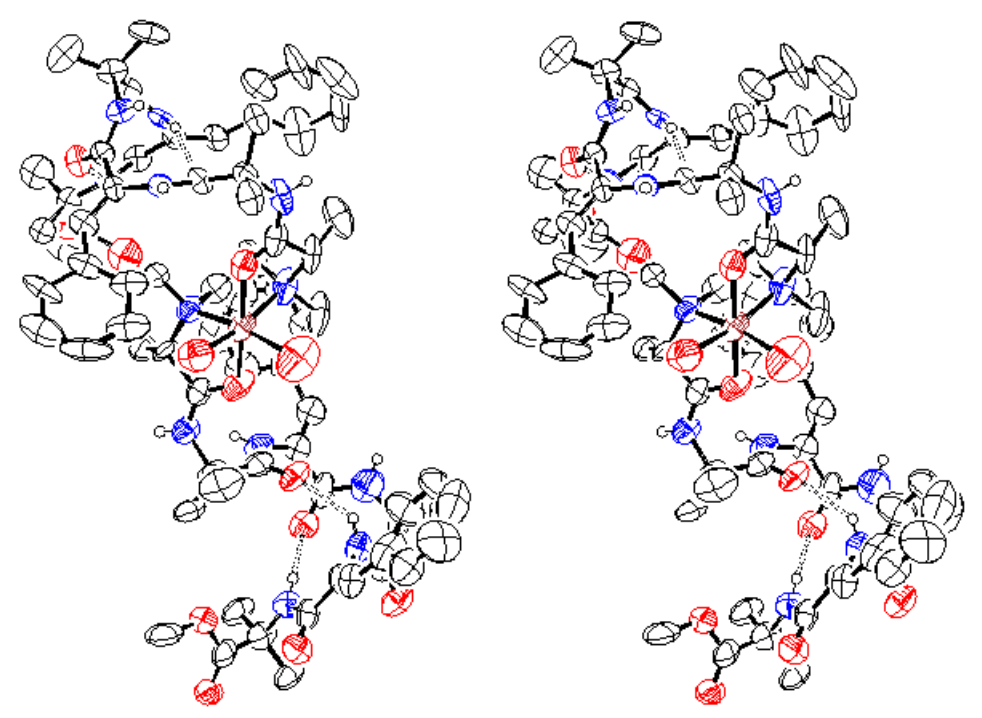

(a)
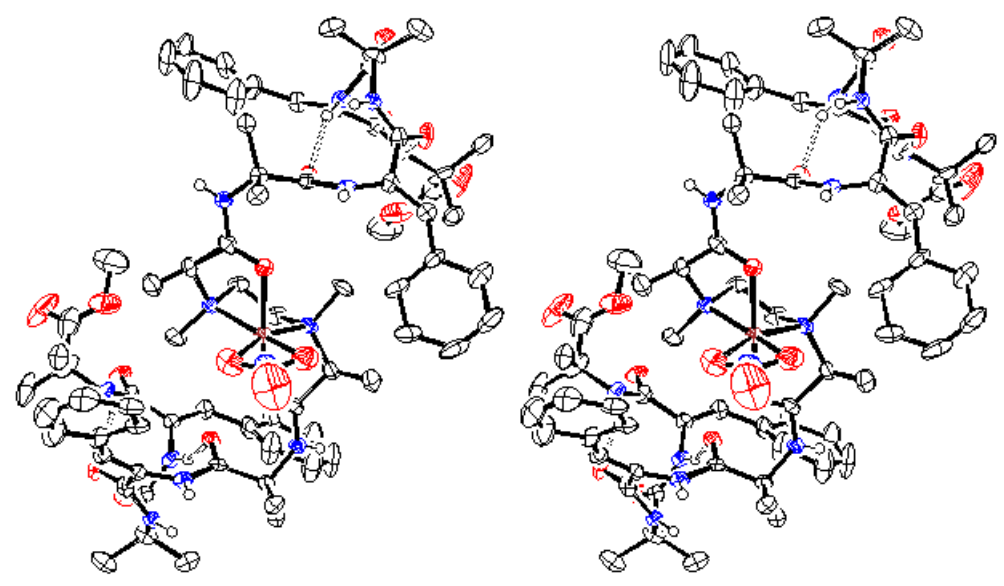

(b)

Figure S5. Stereo view of ORTEP drawing of $\left[\mathrm{Zn}(\mathbf{L 2})\left(\mathrm{H}_{2} \mathrm{O}\right)_{2}\right]\left(\mathrm{ClO}_{4}\right)_{2} \cdot\left(\mathrm{CH}_{3} \mathrm{CN}\right)_{0.5}$. $\left(\mathrm{H}_{2} \mathrm{O}\right)_{10}$ (a) and $\left[\mathrm{Zn}(\mathbf{L 2})\left(\mathrm{NO}_{3}\right)\right]\left(\mathrm{NO}_{3}\right)\left(\mathrm{CH}_{3} \mathrm{CN}\right) \cdot\left(\mathrm{CHCl}_{3}\right)_{5}$ (b) showing $50 \%$ thermal ellipsoids. Solvent molecules, non-coordinating anion and hydrogen atoms except for amide hydrogen atoms are omitted for clarity. 


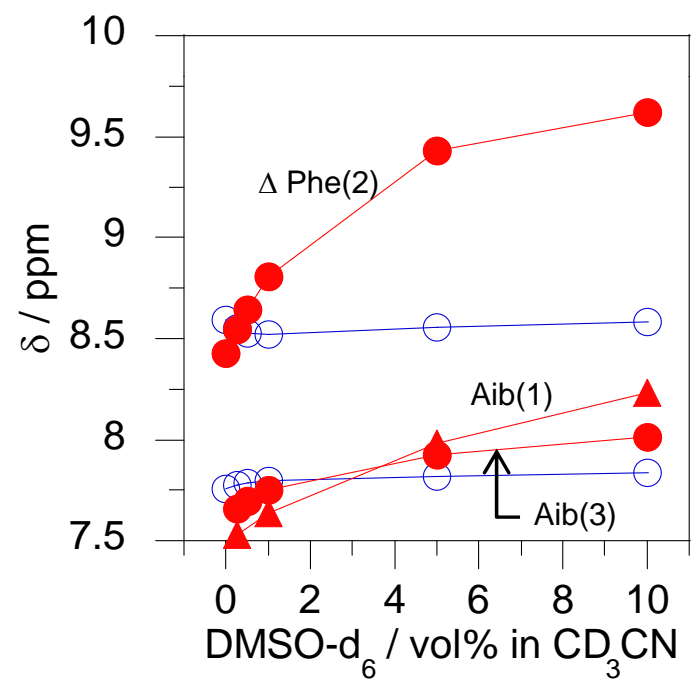

Figure S6. Solvent dependence of $\mathrm{NH}$ chemical shifts of $\mathbf{L 2 - Z n}\left(\mathrm{ClO}_{4}\right)_{2}$ complex in DMSO-d $\mathrm{D}_{6} / \mathrm{CD}_{3} \mathrm{CN}$ mixture system at R.T. [L2] $=\left[\mathrm{Zn}\left(\mathrm{ClO}_{4}\right)_{2} \cdot 6 \mathrm{H}_{2} \mathrm{O}\right]=$ $5 \times 10^{-3} \mathrm{~mol} \mathrm{dm}^{-3}$. 


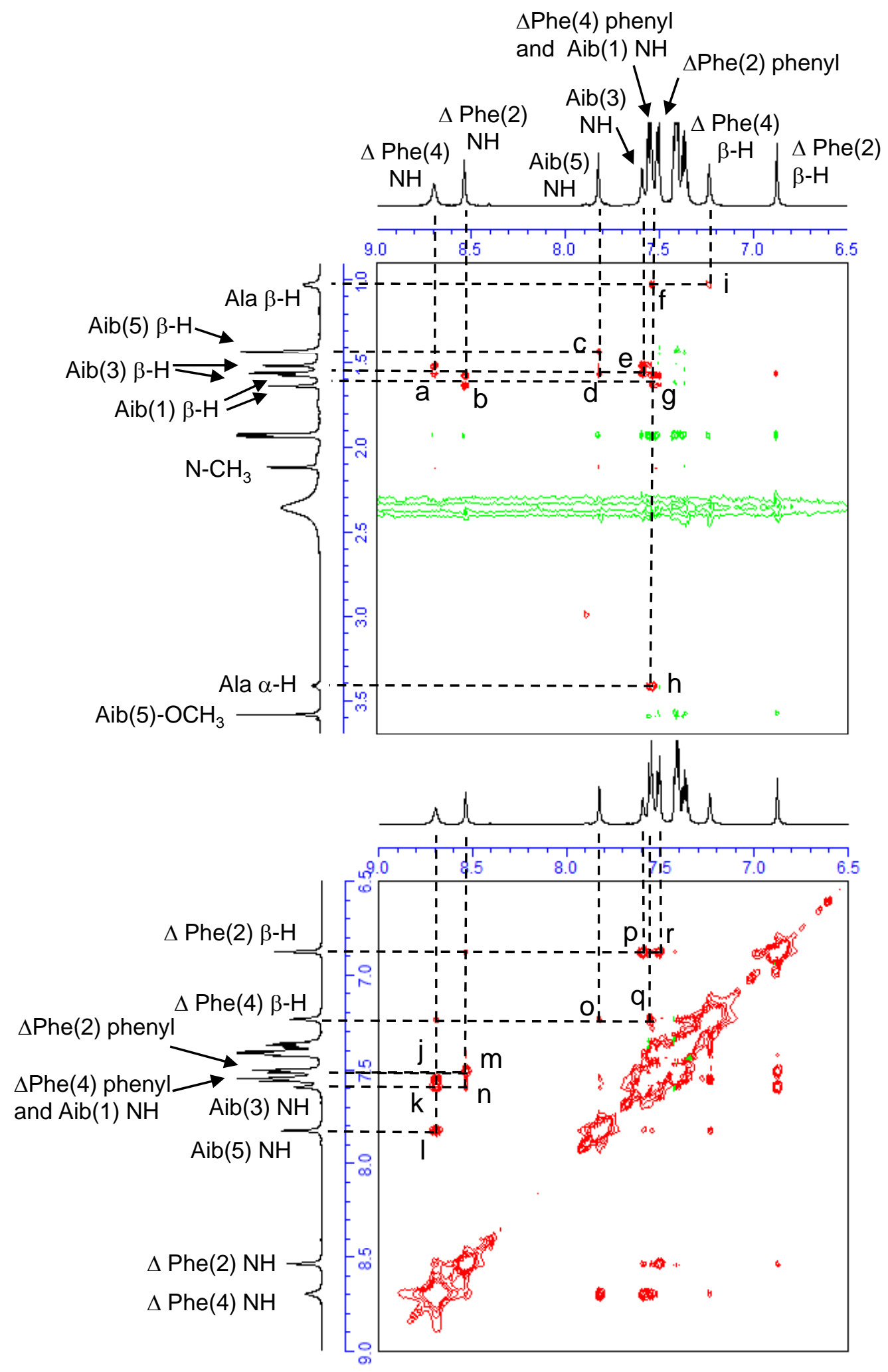

Figure S7. NOESY spectra of $\mathbf{L} 2-\mathrm{Zn}\left(\mathrm{ClO}_{4}\right)_{2}$ complex in $\mathrm{CD}_{3} \mathrm{CN}$ at $-10{ }^{\circ} \mathrm{C}$, $[\mathbf{L 2}]=\left[\mathrm{Zn}\left(\mathrm{ClO}_{4}\right)_{2} \cdot 6 \mathrm{H}_{2} \mathrm{O}\right]=5 \times 10^{-3} \mathrm{~mol} \mathrm{dm}^{-3}$. a: Aib(3) $\beta-\mathrm{H}-\Delta \mathrm{Phe}(4) \mathrm{NH}, \mathrm{b}: \mathrm{Aib}(1) \beta-\mathrm{H}-\Delta \mathrm{Phe}(2) \mathrm{NH}$,

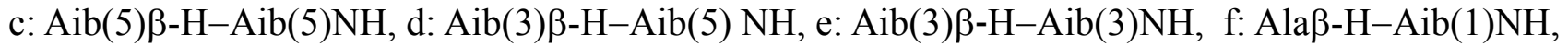

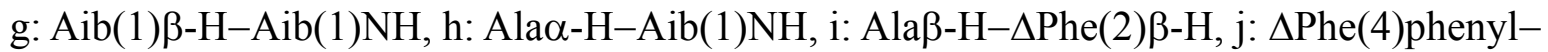
$\Delta \mathrm{Phe}(4) \mathrm{NH}, \mathrm{k}: \mathrm{Aib}(3) \mathrm{NH}-\Delta \mathrm{Phe}(4) \mathrm{NH}, \mathrm{l}$ : Aib(5)NH- $\mathrm{Phe}(4) \mathrm{NH}, \mathrm{m}: \Delta \mathrm{Phe}(2)$ phenyl- $\Delta \mathrm{Phe}(2) \mathrm{NH}$, n: Aib(3)NH- $\Delta$ Phe(2)NH, o: $\Delta \mathrm{Phe}(4) \beta-\mathrm{H}-\mathrm{Aib}(5) \mathrm{NH}, \mathrm{p}: \Delta \mathrm{Phe}(2) \beta-\mathrm{H}-\mathrm{Aib}(3) \mathrm{NH}, \mathrm{q}: \Delta \mathrm{Phe}(4) \beta-\mathrm{H}-$ $\Delta \mathrm{Phe}(4)$ phenyl, r: $\Delta \mathrm{Phe}(2) \beta-\mathrm{H}-\Delta \mathrm{Phe}(2)$ phenyl. 


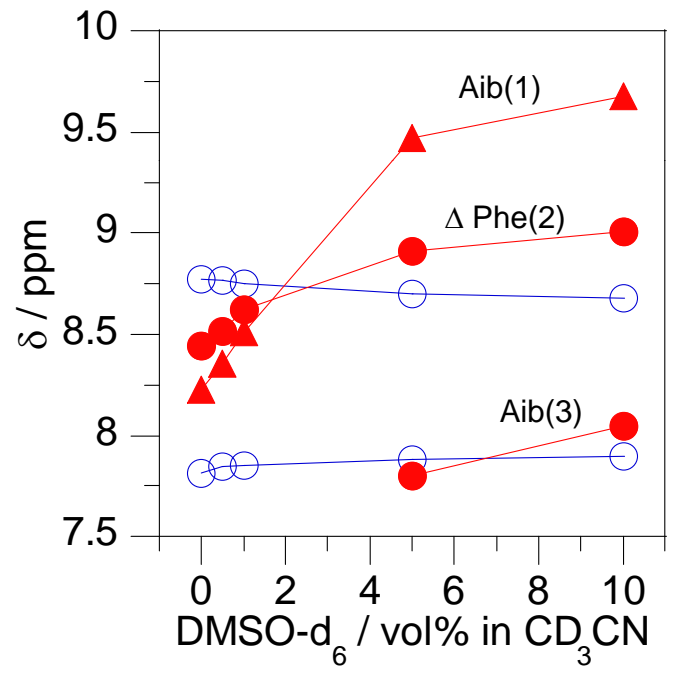

Figure S8. Solvent dependence of $\mathrm{NH}$ chemical shifts of $\mathbf{L 2 - Z n}\left(\mathrm{NO}_{3}\right)_{2}$ complex in DMSO- $\mathrm{d}_{6} / \mathrm{CD}_{3} \mathrm{CN}$ mixture system at R.T. $[\mathbf{L 2}]=\left[\mathrm{Zn}\left(\mathrm{NO}_{3}\right)_{2} \cdot 6 \mathrm{H}_{2} \mathrm{O}\right]=5 \times 10^{-3}$ $\mathrm{mol} \mathrm{dm}{ }^{-3}$. 


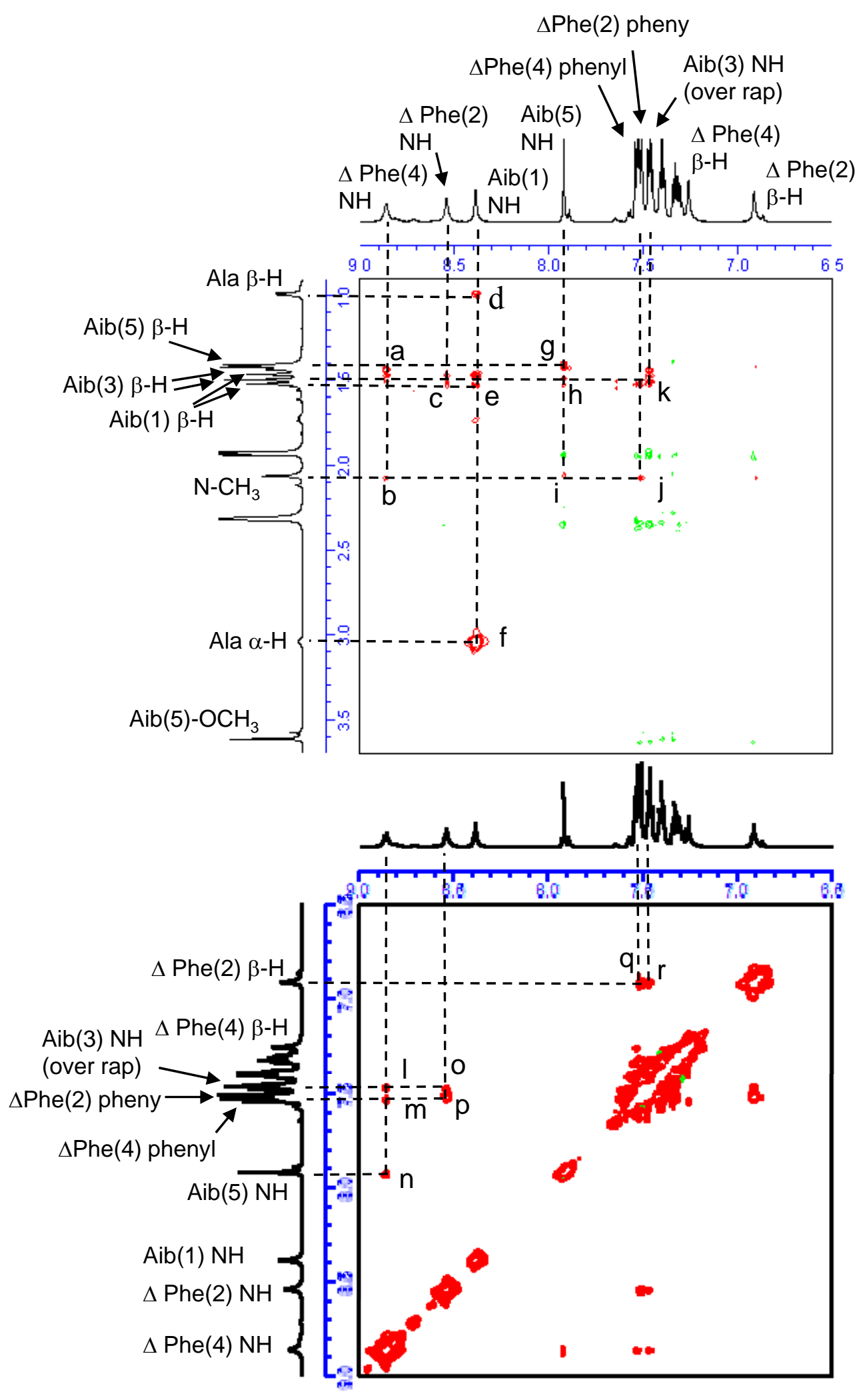

Figure S9. NOESY spectra of $\mathbf{L} 2-\mathrm{Zn}\left(\mathrm{NO}_{3}\right)_{2}$ complex in $\mathrm{CD}_{3} \mathrm{CN}$ at $-10{ }^{\circ} \mathrm{C}$, $[\mathbf{L 2}]=\left[\mathrm{Zn}\left(\mathrm{NO}_{3}\right)_{2} \cdot 6 \mathrm{H}_{2} \mathrm{O}\right]=5 \times 10^{-3} \mathrm{~mol} \mathrm{dm}{ }^{-3} . . \mathrm{a}: \mathrm{Aib}(3) \beta-\mathrm{H}-\Delta \mathrm{Phe}(4) \mathrm{NH}, \mathrm{b}: \mathrm{N}-\mathrm{CH}_{3}-\Delta \mathrm{Phe}(4) \mathrm{NH}$,

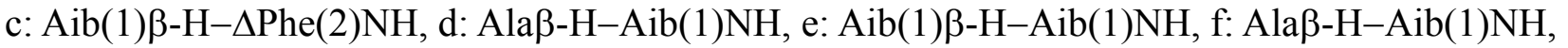
g: $\mathrm{Aib}(5) \beta-\mathrm{H}-\mathrm{Aib}(5) \mathrm{NH}, \mathrm{h}: \mathrm{Aib}(3) \beta-\mathrm{H}-\mathrm{Aib}(5) \mathrm{NH}, \mathrm{i}: \mathrm{N}-\mathrm{CH}_{3}-\mathrm{Aib}(5) \mathrm{NH}, \mathrm{j}: \mathrm{N}-\mathrm{CH}_{3}-$

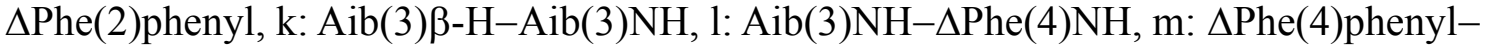
$\Delta \mathrm{Phe}(4) \mathrm{NH}, \mathrm{n}: \mathrm{Aib}(5) \mathrm{NH}-\Delta \mathrm{Phe}(4) \mathrm{NH}, \mathrm{o}: \mathrm{Aib}(3) \mathrm{NH}-\Delta \mathrm{Phe}(2) \mathrm{NH}, \mathrm{p}: \Delta \mathrm{Phe}(2)$ phenyl- $\Delta \mathrm{Phe}(2) \mathrm{NH}$, q: $\Delta \mathrm{Phe}(2) \beta-\mathrm{H}-\Delta \mathrm{Phe}(2)$ phenyl, r: $\Delta \mathrm{Phe}(2) \beta-\mathrm{H}-\mathrm{Aib}(3) \mathrm{NH}$. 
(a)
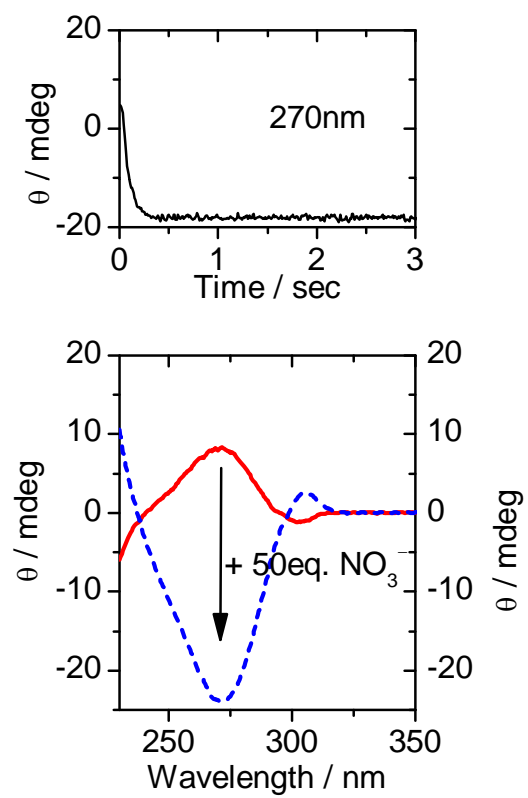

(c)
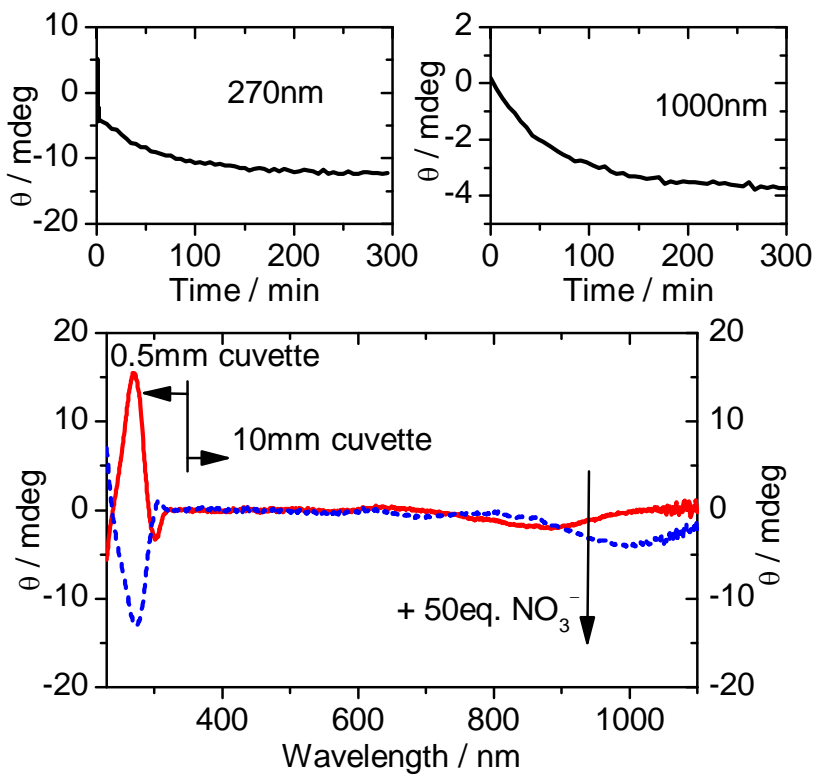

Figure S10. CD spectral changes of $\mathbf{L 2}-\mathrm{M}\left(\mathrm{ClO}_{4}\right)_{2}$ complexes by mixing 50 equiv. of $\mathrm{NO}_{3}{ }^{-}$anion (solid red line $\rightarrow$ dashed blue line) and their relaxation traces in $\mathrm{CH}_{3} \mathrm{CN}$ at R.T. $\{\mathrm{M}=\mathrm{Zn}(\mathrm{a}), \mathrm{Co}(\mathrm{b})$ and $\mathrm{Ni}(\mathrm{c})\}$. [L2] $=0.3 \times 10^{-3} \mathrm{~mol} \mathrm{dm}^{-3}$, $\left[\mathrm{M}\left(\mathrm{ClO}_{4}\right)_{2} \cdot 6 \mathrm{H}_{2} \mathrm{O}\right]=0.6 \times 10^{-3} \mathrm{~mol} \mathrm{dm}^{-3},\left[\mathrm{Bu}_{4} \mathrm{NNO}_{3}\right]=15 \times 10^{-3} \mathrm{~mol} \mathrm{dm}^{-3}$ 
(a)

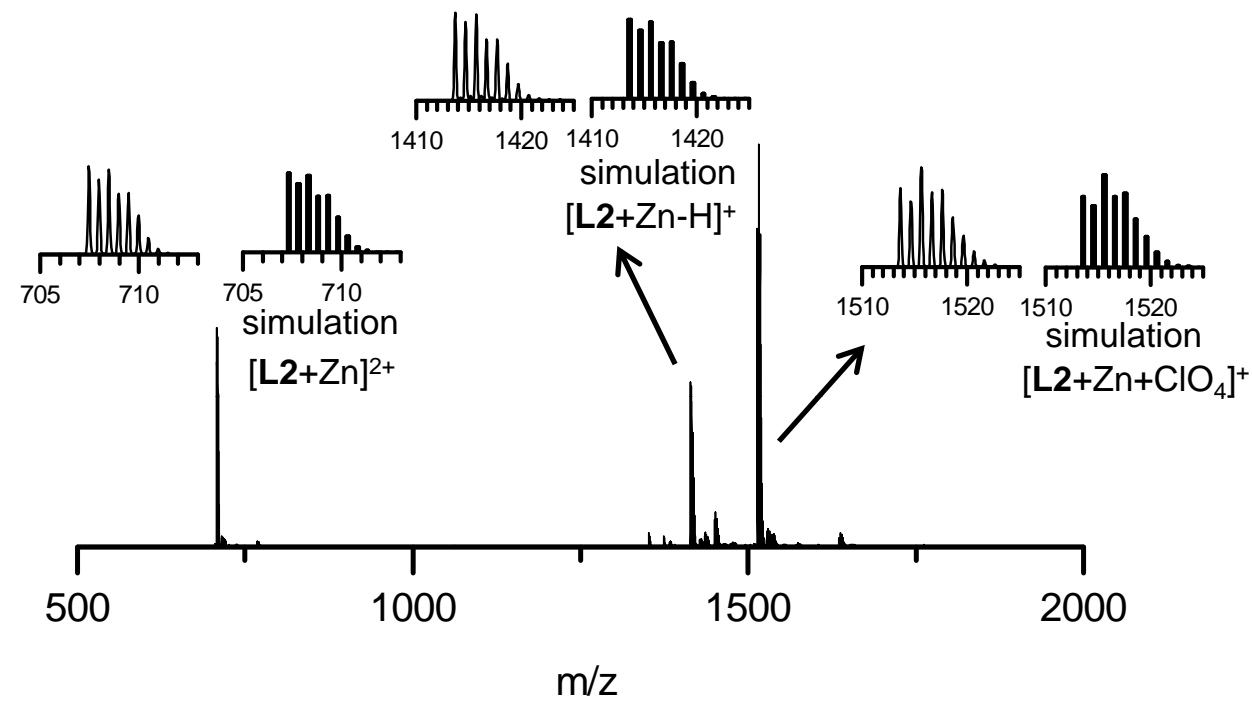

(b)

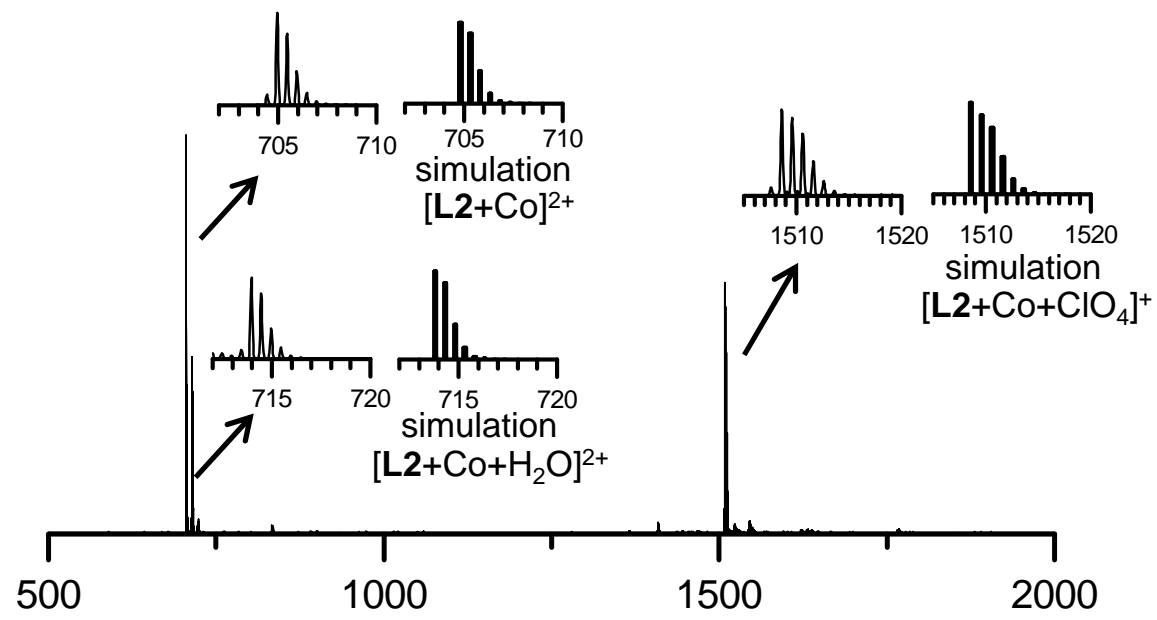

$\mathrm{m} / \mathrm{z}$

(c)

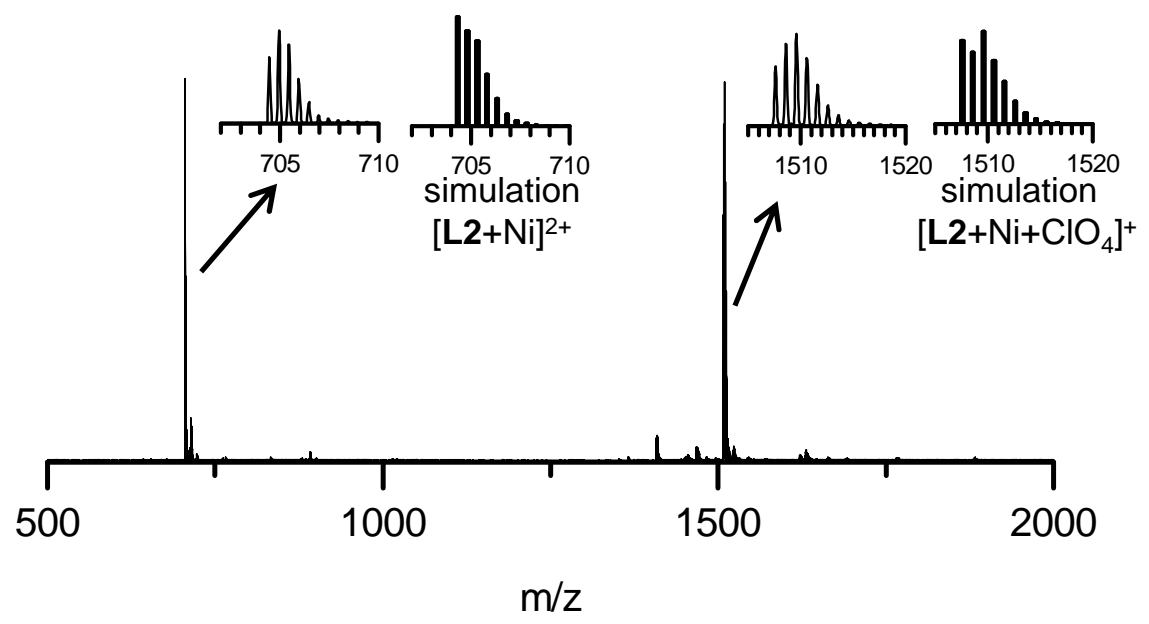

Figure S11. ESI-MS spectra of $\mathbf{L} 2-\mathrm{M}\left(\mathrm{ClO}_{4}\right)_{2}$ complexes in $\mathrm{CH}_{3} \mathrm{CN}$. $\{\mathrm{M}=\mathrm{Zn}(\mathrm{a})$, $\mathrm{Co}$ (b) and Ni (c)\}. 
Table S1. Torsion angles of peptide chains observed in crystal structures of $\mathbf{L} 2-\mathrm{Zn}\left(\mathrm{ClO}_{4}\right)_{2}$ and $-\mathrm{Zn}\left(\mathrm{NO}_{3}\right)_{2}$ complexes.

\begin{tabular}{|c|c|c|c|c|c|c|c|c|c|c|c|}
\hline & \multirow[b]{2}{*}{ chain } & \multirow{2}{*}{$\frac{\text { Ala }}{\psi}$} & \multicolumn{2}{|c|}{$\operatorname{Aib}(1)$} & \multicolumn{2}{|c|}{$\Delta$ Phe(2) } & \multicolumn{2}{|c|}{$\operatorname{Aib}(3)$} & \multicolumn{2}{|c|}{$\Delta$ Phe(4) } & \multirow{2}{*}{$\frac{\operatorname{Aib}(5)}{\phi}$} \\
\hline & & & $\phi$ & $\psi$ & $\phi$ & $\psi$ & $\phi$ & $\psi$ & $\phi$ & $\psi$ & \\
\hline \multirow[t]{2}{*}{$\mathbf{L} 2-\mathrm{Zn}\left(\mathrm{ClO}_{4}\right)_{2}$} & A & -149.7 & -48.3 & 147.8 & -49.3 & -32.8 & -56.6 & -28.0 & -55.0 & -37.4 & 55.0 \\
\hline & B & -176.3 & -55.7 & -50.8 & 44.0 & -126.4 & -56.6 & -27.7 & -63.9 & -17.7 & 53.9 \\
\hline $\mathbf{L} 2-\mathrm{Zn}\left(\mathrm{NO}_{3}\right)_{2}$ & & 153.8 & 56.6 & -141.1 & 52.2 & 24.6 & 49.5 & 35.4 & 69.2 & 30.5 & -31.9 \\
\hline
\end{tabular}

